\title{
Desiccation resistance of wild and mass-reared Bactrocera tryoni (Diptera: Tephritidae)
}

\author{
C.W. Weldon ${ }^{1 *}$, S. Yap ${ }^{2}$ and P.W. Taylor ${ }^{2}$ \\ ${ }^{1}$ Department of Zoology and Entomology, University of Pretoria, \\ Private Bag X20, Hatfield 0028, South Africa: ${ }^{2}$ Department of Biological \\ Sciences, Macquarie University, North Ryde, NSW 2109, Australia
}

\begin{abstract}
In pest management programmes that incorporate the sterile insect technique (SIT), the ability of mass-reared insects to tolerate dry conditions may influence their survival after release in the field. In the present study, desiccation resistance of adult mass-reared Queensland fruit flies, Bactrocera tryoni (Frogatt) (Diptera: Tephritidae), that are routinely released in SIT programmes was compared with that of wild flies at 1,10 and 20 days after adult eclosion. Under dry conditions without access to food or water, longevity of mass-reared B. tryoni was significantly less than that of their wild counterparts. Desiccation resistance of mass-reared flies declined monotonically with age, but this was not the case for wild flies. The sharp decline in desiccation resistance of mass-reared flies as they aged was likely explained by decreased dehydration tolerance. As in an earlier study, desiccation resistance of females was significantly lower than that of males but this was particularly pronounced in mass-reared females. Female susceptibility to dry conditions corresponded with declining dehydration tolerance with age and associated patterns of reproductive development, which suggests that water content of their oocyte load is not available for survival during periods of water stress.
\end{abstract}

Keywords: Body mass, dehydration tolerance, Queensland fruit fly, body water, Tephritidae

(Accepted 17 June 2013; First published online 18 July 2013)

\section{Introduction}

Terrestrial arthropods are particularly vulnerable to water loss because they have a large surface area to volume ratio from which to lose a small volume of body water. Consequently, insects have evolved behavioural, morphological and physiological adaptations that enhance their resistance to desiccation by increasing water storage, restricting water loss or enabling tolerance to dehydration (Chown et al., 2011). Increased desiccation resistance as a consequence of higher

*Author for correspondence

Phone: +27124203234

Fax: +27 123625242

E-mail: cwweldon@zoology.up.ac.za; cwweldon@gmail.com absolute body water content may drive large body size as in desert keratin beetles (Le Lagadec et al., 1998). Improved desiccation resistance in desert Drosophila species is conferred by low rates of respiratory water loss (Gibbs \& Matzkin, 2001) that result from reduced activity (Gibbs et al., 2003). In Drosophila melanogaster, a variety of evolutionary solutions to desiccation stress have been identified in wild and artificially selected populations including high body water content and water storage in the haemolymph (Gibbs et al., 1997; Folk et al., 2001), increased metabolism of stored carbohydrates that produces metabolic water (Marron et al., 2003), enhanced dehydration tolerance and increased mass (Telonis-Scott et al., 2006) and reduction of water loss by increased cuticular melanization (Parkash et al., 2011).

Species differences in desiccation resistance can contribute to distribution and abundance at both large and small scales 
(Le Lagadec et al., 1998; Duyck et al., 2006; Chown et al., 2007; Kellermann et al., 2009; Kleynhans \& Terblanche, 2009; Matzkin et al., 2009). However, desiccation resistance within a species can vary substantially across individuals and populations. Selection can lead to adaptation of populations to dry conditions (Gibbs et al., 1997; Telonis-Scott et al., 2006; Rajpurohit et al., 2008), although this capacity may be limited in some species due to low genetic variation for desiccation resistance traits (Kellermann et al., 2009). Furthermore, events within the life of an individual can induce permanent or reversible changes in structural and functional phenotype that influence subsequent desiccation resistance (Bazinet et al., 2010; Terblanche et al., 2010; Kleynhans \& Terblanche, 2011). For example, larval nutrition can affect desiccation resistance of adult D. melanogaster (Andersen et al., 2010). A short period of water stress can lead to desiccation hardening of adult $D$. melanogaster via a change in cuticular permeability (Bazinet et al., 2010). Agerelated declines in desiccation resistance have been reported in several fly species (Nghiem et al., 2000; Gibbs \& Markow, 2001; Weldon \& Taylor, 2010), with higher water loss rates at older ages identified as the primary cause (Nghiem et al., 2000; Gibbs \& Markow, 2001).

Queensland fruit fly, Bactrocera tryoni (Froggatt) (Diptera: Tephritidae), is the most economically damaging fruit fly species in Australia (Plant Health Australia, 2008). Females can oviposit into ripening fruits of over one hundred plant species, including numerous commercially important crops (Hancock et al., 2000). Developing larvae damage fruit, but also present a serious biosecurity risk (Plant Health Australia, 2008). In some regions, wild B. tryoni populations are managed using area-wide integrated pest management programs that incorporate the sterile insect technique (SIT). SIT is an important method for environmentally benign suppression and eradication of some of the world's most destructive agricultural, horticultural and medical insect pests (see Klassen \& Curtis, 2005). Success of SIT campaigns relies on the ability of sterile males to survive in the field, secure copulations with wild females, induce reproductive sterility in their mates and thereby reduce population levels in the next generation (Knipling, 1959). As SIT requires vast numbers of sterile insects, it is necessary to mass-rear insects under factory conditions that bear little resemblance to their natural environment (Calkins \& Parker, 2005). In the case of $B$. tryoni, the mass-rearing colony is held at a constant temperature $\left(25^{\circ} \mathrm{C}\right)$ and high humidity $(65 \pm 5 \%)$ with constant access to food and water, but SIT releases commonly take place in hot and dry conditions that likely present a major challenge to their survival (Dominiak et al., 2006).

Despite the value of understanding the ability of these serious pests to survive and adapt to environmental conditions when they invade new areas, and climatic envelope modelling suggesting that dry stress plays a major role in determining distribution of B. tryoni (Yonow \& Sutherst, 1998), surprisingly little research has been undertaken to determine their desiccation resistance and tolerance of water loss (Meats, 1989). Weldon \& Taylor (2010) reported that $39 \mathrm{~h}$ was required for $50 \%$ mortality of mass-reared adult $B$. tryoni subjected to a desiccation resistance assay at 1 day after adult eclosion, but by 20 days after adult eclosion, the time required for $50 \%$ mortality had declined significantly to only $29 \mathrm{~h}$. To date, there has been no assessment of desiccation resistance in wild $B$. tryoni, making it difficult to ascertain whether changes induced by mass-rearing represent an increased mortality risk for flies that experience dry conditions. In this study, the assay developed for B. tryoni by Weldon \& Taylor (2010) was used to compare desiccation resistance and tolerance of wild and mass-reared B. tryoni. Furthermore, total body water content and dehydration tolerance of wild and mass-reared $B$. tryon $i$ was compared to elucidate mechanisms that might underlie differences in desiccation resistance.

\section{Materials and methods}

\section{Source of flies and colony maintenance}

Wild $\left(G_{0}\right)$ flies were obtained as larvae in infested fruit (loquat, Eriobotrya japonica) that had been harvested in the Spring of 2009 from trees in the Sydney basin, New South Wales, Australia. Infested fruit were placed on plastic mesh screens positioned over a $20 \mathrm{~mm}$ deep layer of vermiculite (Grade 1, Ausperl, Orica Australia Pty. Ltd, Banksmeadow, NSW, Australia) in a plastic crate. Each crate was enclosed in a mesh cage cover to prevent the escape of 'hopping' larvae. The vermiculite was sifted after 14 days to retrieve the pupae. The pupae were allowed to emerge into a ventilated plastic cage ( 5 litres capacity, $24 \times 15 \times 15 \mathrm{~cm}$ ). On eclosion, 100 adult males and females reared from fruit $\left(G_{0}\right)$ were transferred to separate 5-litre plastic holding cages for use in a desiccation resistance assay (methods described below), and were provided unrestricted access to water and a diet of sucrose and yeast hydrolysate enzymatic (MP Biomedicals, Aurora, $\mathrm{OH}, \mathrm{USA}$ ) in separate dishes.

To obtain $G_{1}$ and $G_{2}$ flies, the remainder of the emerging $G_{0}$ flies (>500 individuals) were housed in a plastic and nylon mesh cage $(47.5 \times 47.5 \times 47.5 \mathrm{~cm}$, BugDorm 4090 Insect Rearing Cage, MegaView Science Co., Taichung, Taiwan) with unrestricted access to water, sucrose and yeast hydrolysate in separate containers. After $G_{0}$ flies had matured sexually (males were observed to call at dusk) they were provided with artificial fruits (c. $30 \mathrm{~mm}$ diameter) of carrot-based larval medium (based on the recipe of Bateman, 1967) wrapped in Parafilm ' $\mathrm{M}$ ' laboratory film (Pechiney Plastic Packaging, Chicago, IL, USA) into which females could oviposit (Kaspi et al., 2001). Artificial fruits were removed after 3-4 days and placed on a $20 \mathrm{~mm}$ deep bed of vermiculite in plastic trays. The vermiculite was sieved gently after 14 days to remove $G_{1}$ pupae. $G_{1}$ pupae were allowed to emerge into a 5-litre cage. On eclosion, $100 G_{1}$ adults were directed to the desiccation resistance assay and the rearing process was repeated with the remaining $G_{1}$ flies (>500 individuals) to obtain $G_{2}$.

Mass-reared B. tryoni were obtained as pupae from a massrearing facility at Elizabeth Macarthur Agricultural Institute (NSW Department of Primary Industries). Adults emerging from irradiated pupae produced by this facility (approximately 5 million per week) are released in SIT campaigns to control B. tryoni outbreaks (Dominiak et al., 2008). The massreared colony was housed in two or three adult cages with approximately 200,000 adults in each cage (Terras et al., 1999), and had been maintained in the facility for over 20 generations at the time of the present study. Pupae were allowed to emerge into a 5-litre cage. On the day of eclosion, 100 adult males and females were transferred to separate 5-litre plastic holding cages for use in a desiccation resistance assay, and were provided with unrestricted access to water and food, as described above. The mass-reared B. tryoni that were used in the desiccation resistance assay had emerged on the same day 
as those from the wild-derived line that were tested. Three batches of mass-reared $B$. tryoni were obtained for the experiment (emerging on 19 October 2009, 6 December 2009 and 1 February 2010). Batches can be regarded as representing consecutive generations from the mass-reared line due to the monthly adult replacement schedule of the mass-rearing facility.

All emergence, holding, and colony cages were maintained, and experiments carried out, at $24-26^{\circ} \mathrm{C}$ and $60-70 \%$ $\mathrm{RH}$ under an LD $14: 10 \mathrm{~h}$ light cycle in a controlled environment room at Macquarie University, Sydney, Australia. The lights were on full intensity for $12 \mathrm{~h}$ and flies also experienced simulated dawn and dusk as the lights stepped on and off in four stages over the course of $1 \mathrm{~h}$.

\section{Desiccation resistance assay}

The protocol used to determine the desiccation resistance of $B$. tryoni with respect to origin, age and sex was based on the assay developed by Gibbs et al. (1997) and has been used previously for B. tryoni (Weldon \& Taylor, 2010). At ages of 1, 10 and 20 days after adult eclosion, 20 wild females and males from each generation were transferred from holding cages to individual $5 \mathrm{ml}$ clear polypropylene flat bottom vials (Techno Plas Pty. Ltd, St. Marys, SA, Australia) that were then loosely stoppered with cotton wool. At the same time, 20 mass-reared females and males of the same age were transferred to individual vials. Flies were then subdued with $\mathrm{CO}_{2}$, weighed (to $0.0001 \mathrm{~g}$ ) using an analytical balance (AB204-S/FACT, Mettler-Toledo $\mathrm{GmbH}$, Greifensee, Switzerland), and returned to their vials, which were once again stoppered with cotton wool. After the initial mass of the flies had been recorded and all flies had recovered, 5-6 large granules of desiccant (6 mesh, Drierite, W.A. Hammond Drierite Co. Ltd, Xenia, $\mathrm{OH}, \mathrm{USA}$ ) were placed into the caps of 80 vials. The caps loaded with desiccant were quickly and tightly screwed onto vials, so that all flies were exposed to desiccating conditions ( $<10 \%$ relative humidity), and a loose barrier of cotton wool separated the flies from the desiccant. Vials were inspected every $3 \mathrm{~h}$ to record mortality. Flies were recorded as dead when no movement of the legs or mouthparts was observed after the vial was gently flicked with a finger. The longevity of each fly under desiccating conditions (desiccation resistance) was calculated. As soon as flies were recorded as dead, their mass at death was recorded before storage in a freezer $\left(-20^{\circ} \mathrm{C}\right)$. All flies were later dried in a drying oven at $50^{\circ} \mathrm{C}$ for $48 \mathrm{~h}$ and weighed a third time (to $0.000001 \mathrm{~g}$ ) with a microbalance (ME5, Sartorius AG, Göttingen, Germany). Dry mass was subtracted from initial weight to calculate total body water content, and from mass at death to calculate body water at death. Body water at death expressed as a percentage of initial body mass is referred to as dehydration tolerance; initial body mass was used as the denominator in this calculation as a proxy for initial body water used by Gibbs et al. (1997) due to a strong positive relationship between these two variables.

\section{Initial water content and metabolic losses of dry mass}

It may be that mass loss by insects during desiccation resistance assays is not only attributable to water loss, but is also in part attributable to reduction of individual mass due to metabolic processes. To gauge the relative contribution of water loss and metabolic loss to mass loss in B. tryoni, the wet mass, dry mass and water content of flies not subjected to a desiccation resistance assay was determined. A separate cohort of wild $\left(G_{0}\right) B$. tryoni were sourced from loquats in the Spring of 2012 following the same methods as outlined above, and mass-reared flies were obtained from the same massrearing facility. Wild and mass-reared adults were allowed to emerge into 5-litre plastic holding cages and were provided unrestricted access to water and a diet of sucrose and yeast hydrolysate enzymatic. At ages of 1,10 and 20 days after adult eclosion, 20 wild and mass-reared $B$. tryoni were randomly selected from holding cages, weighed (to $0.000001 \mathrm{~g}$ ) using a microbalance (fresh mass), and immediately placed in a freezer $\left(-20^{\circ} \mathrm{C}\right)$. At a later date, flies were dried in a drying oven at $50^{\circ} \mathrm{C}$ for $48 \mathrm{~h}$ and weighed again with a microbalance to obtain dry mass.

\section{Data analysis}

To determine the effects of origin, age and sex on desiccation resistance, longevity data were fitted to a parametric survival model assuming a Weibull distribution using PROC LIFEREG in SAS 9.2 (SAS Institute Inc., Cary, NC, USA). Generation is not independent and is strictly a repeated measure of fly origin, but PROC LIFEREG does not accommodate mixed effects models. For this reason, generation and origin were merged into one explanatory variable (origin_gen) with six treatments; wild 0 , wild 1, wild 2, massreared 0 , mass-reared 1 and mass-reared 2 . The initial survival model included origin-gen, age and sex as fixed effects with initial mass as a covariate. The minimum adequate model was then determined by step-wise removal of non-significant terms $(P>0.05)$ from the model. The final fitted model included all predictors and the interaction between origingen and age. Using the final fitted model, estimates and $95 \%$ confidence intervals of median survival time were generated for each combination of treatment effects while controlling for initial mass.

Initial mass, mass at death, dry mass at death and body water at death were fitted to linear mixed-effects models with no autocorrelation structure by maximum likelihood using the 'lme' function from the 'nlme' library in R 2.13.1 ( $R$ Development Core Team, 2007). Dry mass was logtransformed to meet assumptions of normality and homoscedasticity. Explanatory variables in each model were origin, age and sex and their two- and three-way interactions. Generation was incorporated into the model as a repeated measure. In the model explaining water content at death (mg), initial mass (mg) was included as a covariate. Coefficients from each model were used to identify simple effects.

A linear model was constructed in $\mathrm{R}$ to determine the effects of age, sex and origin on the water content of B. tryoni not subjected to a desiccation resistance assay. Fresh mass (mg) was included in the model as a covariate. Inspection of the residuals confirmed that they were normally distributed. Direct statistical comparison of the dry mass of $B$. tryoni before and after desiccation to determine metabolic losses is hampered by the use of two different cohorts. To help to address this problem, the percentage of initial/fresh mass attributable to dry mass without and with desiccation was calculated for $G_{0}$ and mass-reared flies from both cohorts. Another linear model was then used to determine the effect of age, sex, desiccation assay and origin on the percentage of initial/fresh mass attributable to dry mass. The minimal 
Table 1. Summary table for the final fitted parametric survival model that describes desiccation resistance of adult B. tryoni with respect to origin_gen, age and sex. Origin_gen represents the combined effect of fly origin (mass-reared or wild) and generation $(0,1$ or 2$)$.

\begin{tabular}{lrrr}
\hline Predictor & DF & \multicolumn{1}{c}{$\chi^{2}$} & $P$ value \\
\hline Initial mass & 1 & 9.907 & 0.002 \\
Origin_gen & 5 & 143.837 & $<0.001$ \\
Age & 2 & 46.352 & $<0.001$ \\
Sex & 1 & 7.329 & 0.007 \\
Origin_gen $\times$ age & 10 & 70.574 & $<0.001$ \\
\hline
\end{tabular}

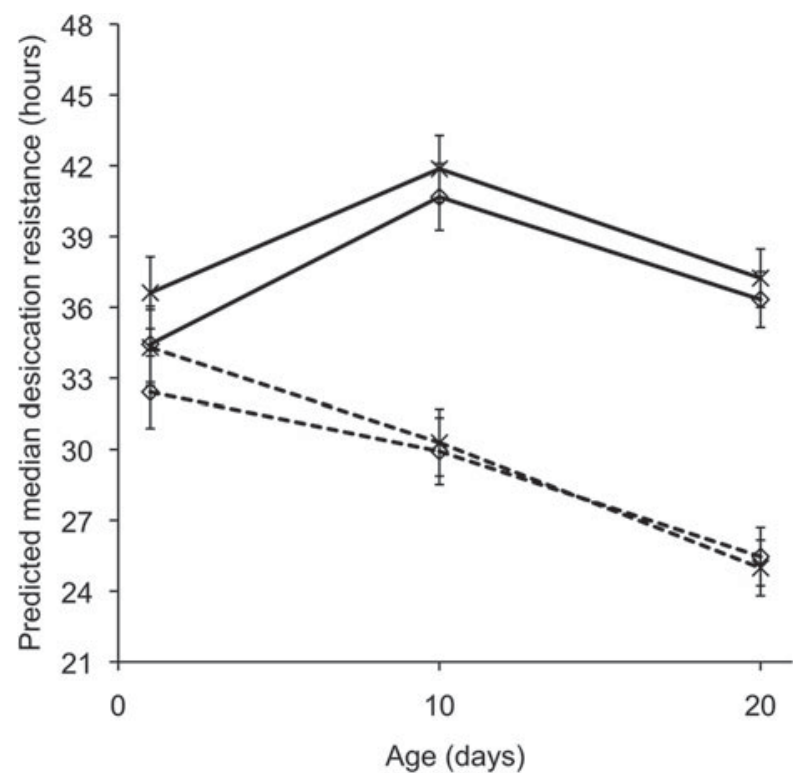

Fig. 1. Median survival time estimated from a parametric survival model describing the survival of adult $B$. tryoni in a desiccation resistance assay with regard to origin gen, age, sex and initial weight. Symbols connected by solid lines are wild adults; symbols connected by dotted lines are mass-reared; diamonds: female, crosses: male. Error bars indicate the upper and lower 95\% confidence interval.

adequate model was then determined using the 'step' function in $R$.

\section{Results}

\section{Desiccation resistance}

Desiccation resistance of $B$. tryoni under dry conditions was significantly affected by initial mass, origin_gen, age, sex and the interaction of origin_gen and age (table 1). Maximumlikelihood parameter estimates from the survival model indicated that desiccation resistance increased with initial mass by a factor of $1.73 \%$. Desiccation resistance of female B. tryoni was $7.03 \%$ less than that of males. Desiccation resistance of all generations of mass-reared B. tryoni declined with age after adult eclosion (fig. 1). There was no effect of age on desiccation resistance in wild $G_{0}$ and $G_{2}$, whereas desiccation resistance in wild $G_{1}$ at 20 days after adult eclosion was significantly less than at 1 and 10 days.
Table 2. Analysis of variance summary tables for the minimum adequate linear mixed-effects models that describe the initial mass, mass at death, dry mass at death and body water at death of adult $B$. tryoni subjected to a desiccation resistance assay with respect to origin, age and sex and their interactions.

\begin{tabular}{|c|c|c|c|c|}
\hline $\begin{array}{l}\text { Dependant } \\
\text { variable }\end{array}$ & Effect & DF & $F$ & value \\
\hline $\begin{array}{l}\text { Initial mass } \\
\quad(\mathrm{mg})\end{array}$ & $\begin{array}{l}\text { (Intercept }) \\
\text { Age } \\
\text { Sex } \\
\text { Origin } \\
\text { Age } \times \text { sex } \\
\text { Age } \times \text { origin } \\
\text { Se } \times \text { origin } \\
\text { Age } \times \text { se } \times \text { origin }\end{array}$ & $\begin{array}{l}1,694 \\
1,694 \\
1,694 \\
1,694 \\
1,694 \\
1,694 \\
1,694 \\
1,694\end{array}$ & $\begin{array}{r}187.606 \\
295.484 \\
2.891 \\
0.708 \\
51.003 \\
6.289 \\
0.281 \\
6.557\end{array}$ & $\begin{array}{r}<0.0001 \\
<\mathbf{0 . 0 0 0 1} \\
0.0895 \\
0.4005 \\
<\mathbf{0 . 0 0 0 1} \\
\mathbf{0 . 0 1 2 4} \\
0.5962 \\
\mathbf{0 . 0 1 0 7}\end{array}$ \\
\hline $\begin{array}{l}\text { Mass at death } \\
\quad(\mathrm{mg})\end{array}$ & $\begin{array}{l}\text { (Intercept }) \\
\text { Age } \\
\text { Sex } \\
\text { Origin } \\
\text { Age } \times \text { sex } \\
\text { Age } \times \text { origin } \\
\text { Se } \times \text { origin } \\
\text { Age } \times \text { se } \times \text { origin }\end{array}$ & $\begin{array}{l}1,694 \\
1,694 \\
1,694 \\
1,694 \\
1,694 \\
1,694 \\
1,694 \\
1,694\end{array}$ & $\begin{array}{r}139.110 \\
397.967 \\
1.731 \\
0.032 \\
76.310 \\
20.160 \\
0.317 \\
17.151\end{array}$ & $\begin{array}{r}<0.0001 \\
<\mathbf{0 . 0 0 0 1} \\
\mathbf{0 . 1 8 8 8} \\
0.8587 \\
<\mathbf{0 . 0 0 0 1} \\
<\mathbf{0 . 0 0 0 1} \\
0.5735 \\
<\mathbf{0 . 0 0 0 1}\end{array}$ \\
\hline $\begin{array}{l}\text { Dry mass at death } \\
\quad\left(\log _{10} \mathrm{mg}\right)\end{array}$ & $\begin{array}{l}\text { (Intercept }) \\
\text { Age } \\
\text { Sex } \\
\text { Origin } \\
\text { Age } \times \text { sex } \\
\text { Age } \times \text { origin } \\
\text { Se } \times \text { origin } \\
\text { Age } \times \text { se } \times \text { origin }\end{array}$ & $\begin{array}{l}1,694 \\
1,694 \\
1,694 \\
1,694 \\
1,694 \\
1,694 \\
1,694 \\
1,694\end{array}$ & $\begin{array}{r}117.157 \\
416.498 \\
0.870 \\
5.037 \\
60.239 \\
7.876 \\
0.845 \\
15.455\end{array}$ & $\begin{array}{r}<0.0001 \\
<0.0001 \\
0.6311 \\
\mathbf{0 . 0 2 5 1} \\
<\mathbf{0 . 0 0 0 1} \\
\mathbf{0 . 0 0 5 1} \\
0.3582 \\
\mathbf{0 . 0 0 0 1}\end{array}$ \\
\hline $\begin{array}{l}\text { Body water at } \\
\text { death (mg) }\end{array}$ & $\begin{array}{l}\text { (Intercept) } \\
\text { Age } \\
\text { Sex } \\
\text { Origin } \\
\text { Initial mass }(\mathrm{mg}) \\
\text { Age } \times \text { sex } \\
\text { Age } \times \text { origin } \\
\text { Sex } \times \text { origin } \\
\text { Age } \times \text { sex } \times \text { origin }\end{array}$ & $\begin{array}{l}1,693 \\
1,693 \\
1,693 \\
1,693 \\
1,693 \\
1,693 \\
1,693 \\
1,693 \\
1,693\end{array}$ & $\begin{array}{r}6.738 \\
22.066 \\
1.088 \\
3.518 \\
581.411 \\
6.593 \\
25.492 \\
0.343 \\
5.147\end{array}$ & $\begin{array}{r}0.0096 \\
<\mathbf{0 . 0 0 0 1} \\
0.2973 \\
0.0611 \\
<\mathbf{0 . 0 0 0 1} \\
\mathbf{0 . 0 1 0 4} \\
<\mathbf{0 . 0 0 0 1} \\
0.5585 \\
\mathbf{0 . 0 2 3 6}\end{array}$ \\
\hline
\end{tabular}

\section{Body mass and water content}

Initial mass varied significantly with the interaction of age, sex and origin (table 2). At 1 day after adult eclosion, within each origin females and males had similar initial mass, but wild flies were significantly heavier than their mass-reared counterparts (fig. 2). Thereafter, initial mass of females increased to be greater than that of males by 10 days after adult eclosion. Reversing the pattern found at 1 day after adult eclosion, initial mass of mass-reared females was greater than that of wild females at this age. By 20 days after adult eclosion, mass-reared and wild females had similar initial mass and had significantly greater mass than males (fig. 2). Throughout the experiment, the initial mass of wild males was greater than that of massreared males (fig. 2).

The interaction of age, sex and origin had a significant effect on mass at death (table 2). Females had greater mass at death than males. Also, mass-reared females had greater mass at death than wild females, particularly at 10 and 20 days after adult eclosion (fig. 2). Wild and mass-reared males had similar mass at death at 1 and 10 days after adult eclosion, but by 20 days, mass-reared males had lower body mass at death than wild males (fig. 2). 

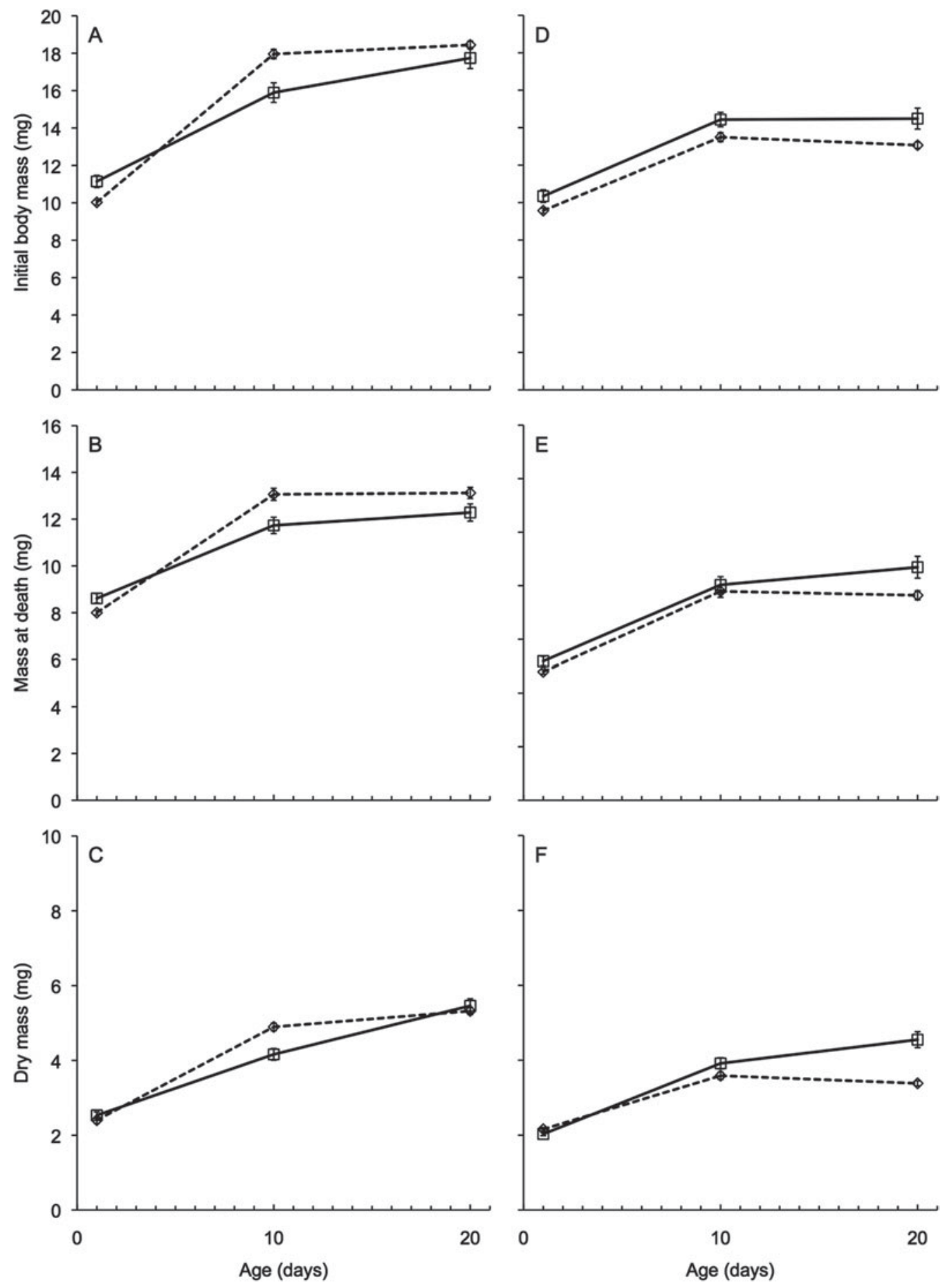

Fig. 2. Mean initial body mass, mass at death and dry mass at death of adult $B$. tryoni in relation to origin, age and sex. Females: initial body mass (A), mass at death (B), dry mass at death (C). Males: initial body mass (D), mass at death (E), dry mass at death (F). Squares connected by solid lines are wild adults; diamonds connected by dotted lines are mass-reared adults. Error bars indicate 1 SE.

Dry mass at death was significantly affected by the interaction of age, sex and origin (table 2). Females consistently had greater dry mass than males (fig. 2). At 1 day after adult eclosion, mass-reared and wild flies of each sex had similar dry mass. Ten days after adult eclosion dry mass of mass-reared females was greater than that of wild females, but by 20 days after adult eclosion dry mass of mass-reared and wild females was similar and significantly greater than that of males (fig. 2). Dry mass of wild males increased throughout the experiment and was greater than 

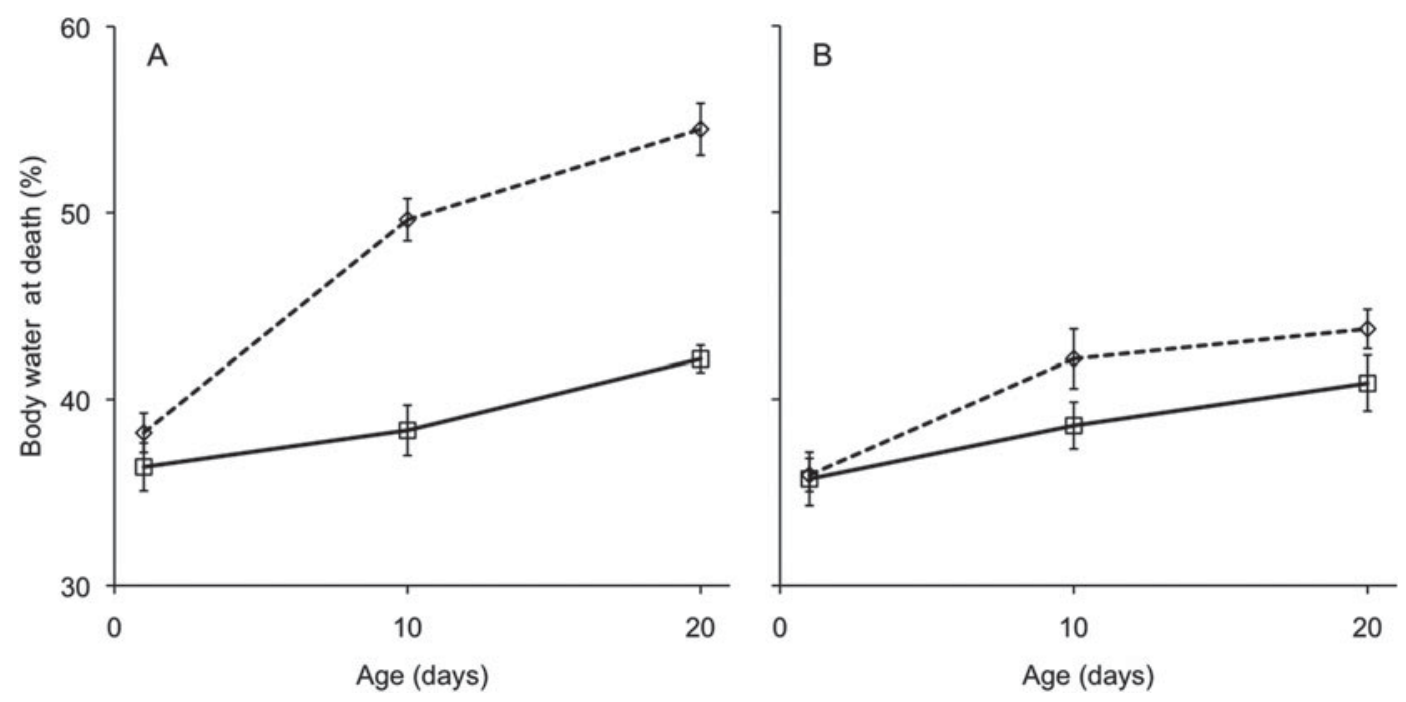

Fig. 3. Mean dehydration tolerance of adult female (A) and male (B) B. tryoni with respect to origin, age and sex. Dehydration tolerance is body water at death $(\mathrm{mg})$ divided by initial body mass $(\mathrm{mg})$. Squares connected by solid lines are wild adults; diamonds connected by dotted lines are mass-reared adults. Error bars indicate $1 \mathrm{SE}$.

that of mass-reared males at 10 and 20 days after adult eclosion (fig. 2).

\section{Dehydration tolerance}

Body water at death was significantly affected by initial mass (table 2), increasing significantly as initial mass increased (body water at death $=-0.59+0.36 \times$ initial mass). When accounting for total body water, the interaction of age, sex and origin significantly affected the amount of body water at death (table 2). When tested at 1 day after adult eclosion, body water at death was similar regardless of sex or origin (fig. 3). Thereafter, body water at death increased with age in both sexes of mass-reared and wild flies. However, by 20 days after adult eclosion, body water at death of mass-reared females was higher than that of all other groups (fig. 3). Also by 20 days after adult eclosion, the body water at death of mass-reared males was higher than that of wild females and wild males when accounting for total body water (fig. 3).

\section{Initial water content and metabolic losses of dry mass}

The pattern of fresh mass (fig. 4) of unstressed B. tryoni with respect to age, sex and origin was largely similar to the initial mass of those that were desiccated (fig. 2). The fresh mass of unstressed flies was described by the main effects of age, sex and origin (table 3). In this cohort, fresh mass increased significantly with age, females were significantly heavier than males and mass-reared flies were significantly heavier than their wild counterparts (fig. 4). The water content of unstressed B. tryoni was affected significantly by the main effects of age and fresh mass (table 3). Water content increased with age (fig. 4) and this was accompanied by a significant, positive relationship between fresh mass and water content (water content $=0.14+$ $0.61 \times$ fresh mass, $R^{2}=0.95$; table 3 ).

The interactions of stress with age, and age with origin significantly affected the contribution of dry mass to
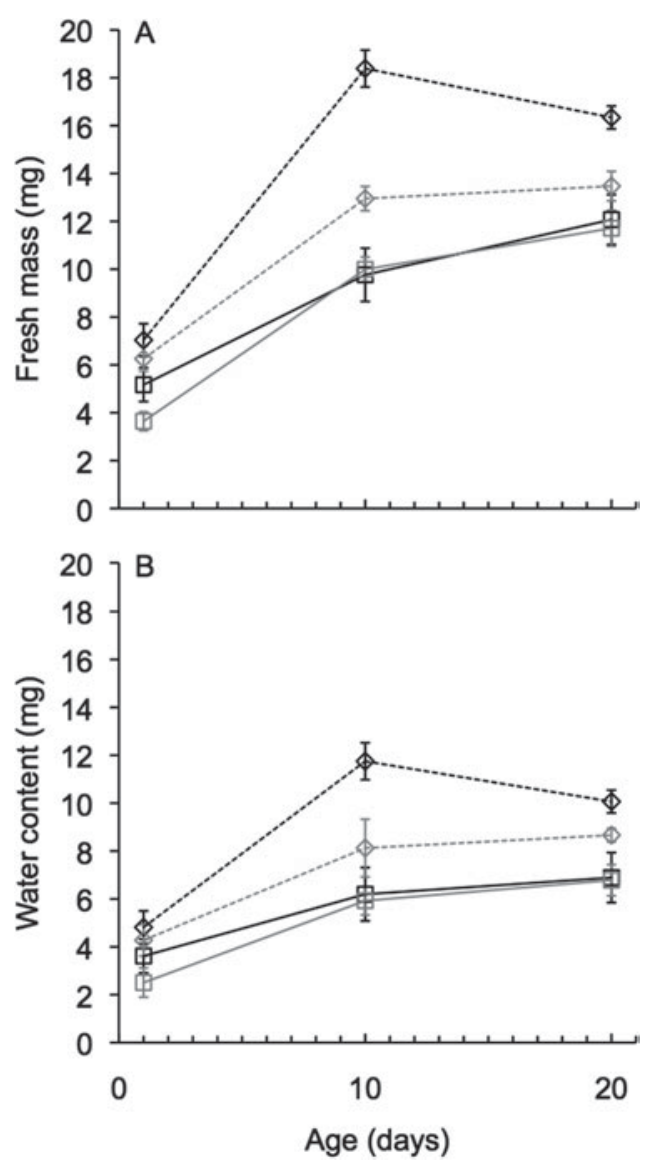

Fig. 4. Mean fresh mass (A) and water content (B) of adult B. tryoni in relation to origin, age and sex. Squares connected by solid lines are wild; diamonds connected by dotted lines are mass-reared. Black markers and lines are females; grey markers and lines are males. Error bars indicate $1 \mathrm{SE}$. 
Table 3. Analysis of variance table for the linear model that describes fresh mass and water content of adult $B$. tryoni with respect to age, sex and origin $\left(G_{0}\right.$ or mass-reared). Fresh mass was included as a covariate in the linear model for water content to account for the effect of individual size.

\begin{tabular}{llrrr}
\hline $\begin{array}{l}\text { Dependant } \\
\text { variable }\end{array}$ & \multicolumn{1}{c}{ Predictor } & DF & \multicolumn{1}{c}{$F$} & $P$ value \\
\hline Fresh mass (mg) & Intercept & 1,112 & 126.324 & $<0.0001$ \\
& Age & 1,112 & 42.288 & $<\mathbf{0 . 0 0 0 1}$ \\
& Sex & 1,112 & 6.351 & $\mathbf{0 . 0 1 3 1}$ \\
& Origin & 1,112 & 12.287 & $\mathbf{0 . 0 0 0 7}$ \\
& Age $\times$ sex & 1,112 & 0.862 & 0.3551 \\
& Age $\times$ origin & 1,112 & 0.927 & 0.3376 \\
& Sex $\times$ origin & 1,112 & 1.548 & 0.2160 \\
Water content & Age $\times$ sex $\times$ origin & 1,112 & 1.007 & 0.3178 \\
Img) & Intercept & 1,111 & 0.928 & 0.3374 \\
& Age & 1,111 & 4.612 & $\mathbf{0 . 0 3 3 9}$ \\
& Sex & 1,111 & 0.196 & 0.6588 \\
& Origin & 1,111 & 0.039 & 0.8446 \\
& Fresh mass (mg) & 1,111 & 1001.106 & $<\mathbf{0 . 0 0 0 1}$ \\
& Age $\times$ sex & 1,111 & 1.469 & 0.2281 \\
& Age $\times$ origin & 1,111 & 0.852 & 0.3581 \\
& Sex $\times$ origin & 1,111 & 0.150 & 0.6995 \\
& Age $\times$ sex $\times$ origin & 1,111 & 0.170 & 0.6837 \\
\hline
\end{tabular}

initial/fresh mass (table 4). The percentage of dry mass in unstressed B. tryoni was higher than their desiccated counterparts, but this difference declined with age as the dry mass remaining in desiccated flies was greatest at 20 days after eclosion (fig. 5). In both unstressed and desiccated flies, the dry mass contribution to initial/fresh mass was similar in wild and mass-reared flies at younger ages, but by 20 days after adult eclosion the percentage of dry mass in wild flies was greater than that of mass-reared flies (fig. 5).

\section{Discussion}

In this study, mass-reared and wild B. tryoni were found to differ in desiccation resistance; under dry conditions without access to food or water, longevity of mass-reared B. tryoni was significantly shorter than that of their wild counterparts (fig. 1). Mass-reared flies that are released for SIT during dry conditions will likely not survive more than two days, even when provided with food and water prior to release, unless they are able to locate protective microhabitats and water in the field. It is unlikely that this result is due to differences in response to starvation because median survival of massreared flies without access to food is approximately $10 \mathrm{~h}$ longer than those that are starved and held under dry conditions (Weldon \& Taylor, 2010). The wild flies used in this study were sourced from a coastal, humid area. It has been suggested that some $B$. tryoni populations may be adapted to local climatic conditions (Bateman, 1967), so the difference in desiccation resistance between wild and mass-reared $B$. tryoni may be conservative in comparison to wild flies from drier inland regions. In general, there was a slight increase in desiccation resistance with initial mass of $B$. tryoni. This finding agrees with the principle proposed by Dominiak et al. (2002) that 'bigger is better' for mass-reared insects used in SIT programmes, but as discussed below, older mass-reared flies with a high initial mass may not necessarily exhibit high desiccation resistance.
Table 4. Minimal adequate linear model that describes the percentage of initial/fresh mass attributable to dry mass of adult $B$. tryoni with respect to desiccation assay (not tested or tested), age, sex and origin $\left(G_{0}\right.$ or mass-reared).

\begin{tabular}{lccr}
\hline Predictor & DF & $F$ & $P$ value \\
\hline Intercept & 1,354 & 444.435 & $<0.0001$ \\
Assay & 1,354 & 131.842 & $<\mathbf{0 . 0 0 0 1}$ \\
Age & 1,354 & 13.950 & $\mathbf{0 . 0 0 0 2}$ \\
Origin & 1,354 & 0.1282 & 0.7205 \\
Assay $\times$ age & 1,354 & 5.690 & $\mathbf{0 . 0 1 7 6}$ \\
Origin $\times$ age & 1,354 & 4.722 & $\mathbf{0 . 0 3 0 4}$ \\
\hline
\end{tabular}

Age had a particularly pronounced effect on desiccation resistance of mass-reared $B$. tryoni relative to their wild counterparts. As in an earlier study of mass-reared B. tryoni (Weldon \& Taylor, 2010), desiccation resistance declined with age. In the present study, median desiccation resistance of mass-reared female and male $B$. tryoni was only $25 \mathrm{~h}$ when tested at 20 days after adult eclosion. The present results for $B$. tryoni are similar to those found in Drosophila; in both mesic and xeric Drosophila species, desiccation resistance declines with age (Gibbs \& Markow, 2001). The age-related decline in desiccation resistance found in Drosophila coincides with increased rates of water loss (Nghiem et al., 2000; Gibbs \& Markow, 2001). In B. tryoni, measurements of initial mass and dry mass provide little explanation for the sharp decline in desiccation resistance of mass-reared flies as they age. However, when accounting for initial mass (which is strongly correlated with water content), both female and male massreared $B$. tryoni contained more body water at death (i.e. had lower dehydration tolerance) than their wild counterparts at 10 and 20 days after adult eclosion. The mechanisms that confer dehydration tolerance in non-dormant insects may involve compartmentalization of water, regulation of osmotic effects and the rendering of a proportion of water osmotically inactive (Danks, 2000). In D. melanogaster, increased desiccation resistance in laboratory-selected lines has been associated with increased carbohydrate content (Djawdan et al., 1998). Subsequent studies have suggested that intracellular glycogen storage and accumulation of trehalose in the haemolymph likely act to retain water in intracellular and body fluids due to molecular binding and osmotic effects (Folk et al., 2001; Gefen et al., 2006). Regulation of sodium, chloride and potassium ion levels in the haemolymph also serves to reduce water volume loss by an osmotic effect (Folk \& Bradley, 2003). In another tephritid fly, Ceratitis capitata, carbohydrates represent the majority of stored macronutrients in field-caught males (Warburg \& Yuval, 1997; Yuval et al., 1998), but there are no published reports that compare whole body or haemolymph carbohydrate or ion concentrations in wild and mass-reared tephritids, so it is too early to suggest a mechanism that confers the diminished dehydration tolerance of mass-reared B. tryoni.

In both mass-reared and wild B. tryoni, females had lower desiccation resistance than males. This result aligns well with an earlier study on mass-reared B. tryoni (Weldon \& Taylor, 2010). However, in most species of Drosophila that have been studied, females exhibit similar or higher desiccation resistance than males (Gibbs \& Markow, 2001; Matzkin et al., 2009). In D. melanogaster, high female resistance to desiccation relative to males is associated with higher body water content, 

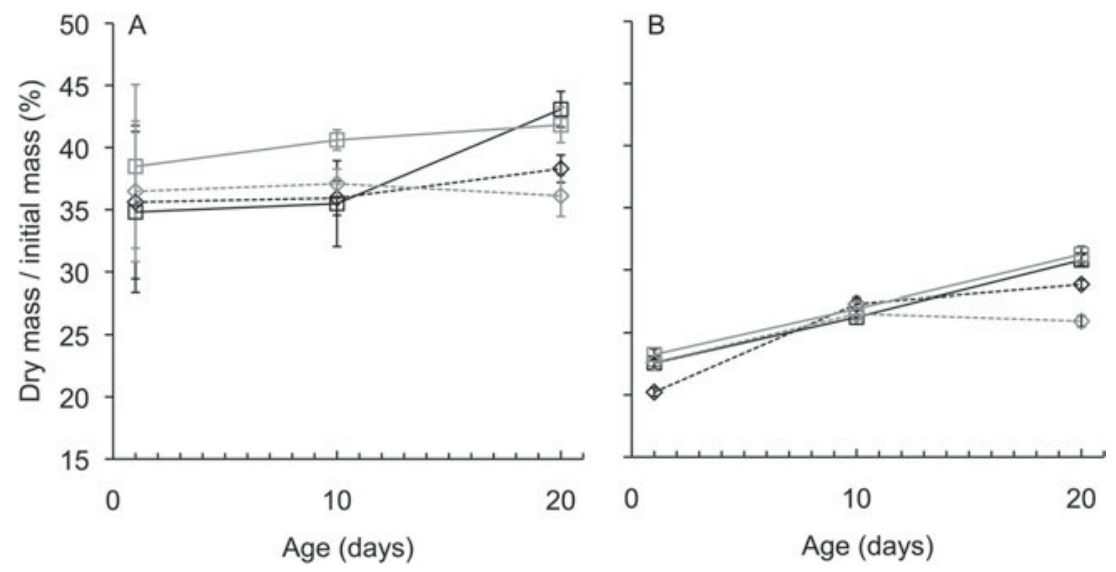

Fig. 5. Mean percentage of initial or fresh mass accounted for by dry mass in unstressed (A) and desiccated (B) adult B. tryoni in relation to origin, age and sex. Squares connected by solid lines are wild; diamonds connected by dotted lines are mass-reared. Black markers and lines are females; grey markers and lines are males. Error bars indicate 1 SE.

as well as a higher percentage of longer chain length cuticular hydrocarbons with higher melting points that provide a more effective barrier to cuticular water loss (Gibbs et al., 1997). Why is it that female B. tryoni suffer from desiccation more than males? Female B. tryoni gained mass as they aged as a consequence of both increased dry mass (fig. 2) and body water content (fig. 3). Mass-reared females gained mass at a faster rate than wild females and males of both origins, which is not unexpected due to their rapid sexual development and large reproductive investment (Meats et al., 2004). By 10 days after adult eclosion, mass-reared female $B$. tryoni with access to a nutrient-rich diet have reached sexual and reproductive maturity and carry large numbers of eggs in their ovaries (Perez-Staples et al., 2007; Pérez-Staples et al., 2011). In contrast, reproductive maturity of wild females is reached between 15 and 20 days after adult eclosion and mature oocyte load is consistently lower than that of laboratory-adapted females (Meats et al., 2004). At the same ages when massreared and wild females are expected to be reproductively mature their mass at death was at its peak and dehydration tolerance declined. Consequently, in female B. tryoni it seems likely that body water is partitioned between the female soma and her oocytes. The lower dehydration tolerance of massreared female $B$. tryoni suggests that water content of their large oocyte load is not available to females for survival during periods of water stress.

During desiccation resistance assays, B. tryoni lose a considerable amount of dry mass. This is probably a result of the use of energy reserves to support metabolism and to confer some level of desiccation resistance. Some of the literature on desiccation resistance that use time-to-death assays and measure water loss gravimetrically is based on an assumption that mass loss during these assays is the result of water loss only (Coenen-Staß, 1986; Bahrndorff et al., 2006; Telonis-Scott et al., 2006; Andersen et al., 2010; Bazinet et al., 2010). The results of this study support those of Folk \& Bradley $(2003,2004)$ that mass loss of insects during desiccation results from a loss of both water and dry mass. The percentage of dry mass relative to initial/fresh mass in desiccated $B$. tryoni increased with age, which is likely due to the shorter time span that these animals must rely on their metabolic reserves before death.
Improving the desiccation resistance of mass-reared B. tryoni that are produced for release in SIT programmes may contribute to improved control outcomes. It has been proposed that understanding physiological adaptation of mass-reared insects to environmental conditions, and manipulating rearing or holding conditions prior to release can improve field performance (Sørensen et al., 2012). Phenotypic plasticity within the adult life stage of $B$. tryoni is currently not known, but the results of Bazinet et al. (2010) offer the possibility that desiccation resistance of mass-reared adults could be improved by a conditioning period of water stress prior to release. Alternatively, it may be necessary to alter the holding conditions of the mass-reared colony to select for traits that confer improved desiccation resistance (as reviewed by Sørensen et al., 2012). However, both of these approaches need to be preceded by further studies to establish the mechanisms that confer desiccation resistance and dehydration tolerance in B. tryoni, their heritability, and their plasticity in response to developmental history and environmental conditions.

\section{Acknowledgements}

The authors thank John Prenter and Anne Wignall for assistance with the setting up of the desiccation resistance assays and for helping to monitor them. We are also indebted to Sam Collins and Rowan McGinley for collecting infested fruit and weighing flies. Maria Castillo-Pando assisted CWW with maintenance of the wild fly colony. Staff at NSW Department of Primary Industries, especially Laura Jiang and Selliah Sundaralingam, provided pupae generously from the mass-production facility at the Elizabeth Macarthur Agricultural Institute. John Terblanche (Stellenbosch University, South Africa) provided valuable comments on a draft version of the manuscript. This project was facilitated by Horticulture Australia Limited (HAL) in partnership with Australian Citrus Growers and was funded by the Citrus levy (Project code CT07036). The Australian Government provides matched funding for all HAL R\&D activities. 


\section{References}

Andersen, L.H., Kristensen, T.N., Loeschcke, V., Toft, S. \& Mayntz, D. (2010) Protein and carbohydrate composition of larval food affects tolerance to thermal stress and desiccation in adult Drosophila melanogaster. Journal of Insect Physiology 56, 336-340.

Bahrndorff, S., Holmstrup, M., Petersen, H. \& Loeschcke, V. (2006) Geographic variation for climatic stress resistance traits in the springtail Orchesella cincta. Journal of Insect Physiology 52, 951-959.

Bateman, M.A. (1967) Adaptations to temperature in geographic races of the Queensland fruit fly, Dacus (Strumeta) tryoni (Froggatt). Australian Journal of Zoology 15, 1141-1161.

Bazinet, A.L., Marshall, K.E., Macmillan, H.A., Williams, C.M. \& Sinclair, B.J. (2010) Rapid changes in desiccation resistance in Drosophila melanogaster are facilitated by changes in cuticular permeability. Journal of Insect Physiology 56, 2007-2013.

Calkins, C.O. \& Parker, A.G. (2005) Sterile insect quality. pp. 269-296 in Dyck, V.A., Hendrichs, J. \& Robinson, A.S. (Eds) Sterile Insect Technique: Principles and Practice in AreaWide Integrated Pest Management. Dordrecht, Netherlands, Springer.

Chown, S.L., Slabber, S., McGeoch, M.A., Janion, C. \& Leinaas, H.P. (2007) Phenotypic plasticity mediates climate change responses among invasive and indigenous arthropods. Proceedings of the Royal Society B 274, 2531-2537.

Chown, S.L., Sørensen, J.G. \& Terblanche, J.S. (2011) Water loss in insects: an environmental change perspective. Journal of Insect Physiology 57, 1070-1084.

Coenen-Staß, D. (1986) Investigations on the water balance in the red wood ant, Formica polyctena (Hymenoptera, Formicidae): workers, their larvae and pupae. Comparative Biochemistry and Physiology 83A, 141-147.

Danks, H.V. (2000) Dehydration in dormant insects. Journal of Insect Physiology 46, 837-852.

Djawdan, M., Chippindale, A.K., Rose, M.R. \& Bradley, T.J. (1998) Metabolic reserves and evolved stress resistance in Drosophila melanogaster. Physiological Zoology 71, 584-594.

Dominiak, B.C., Sundaralingam, S., Jessup, A.J. \& Barchia, I.M. (2002) Pupal weight as a key indicator for quality of mass produced adult Queensland fruit fly Bactrocera tryoni (Froggatt) (Diptera: Tephritidae) in 1997/98. General and Applied Entomology 31, 17-24.

Dominiak, B.C., Mavi, H.S. \& Nicol, H.I. (2006) Effect of town microclimate on the Queensland fruit fly Bactrocera tryoni. Australian Journal of Experimental Agriculture 46, 1239-1249.

Dominiak, B.C., Sundaralingam, S., Jiang, L., Jessup, A.J. \& Barchia, I.M. (2008) Production levels and life history traits of mass-reared Queensland fruit fly Bactrocera tryoni (Froggatt) (Diptera: Tephritidae) during 1999/2002 in Australia. Plant Protection Quarterly 23, 131-135.

Duyck, P.-F., David, P. \& Quilici, S. (2006) Climatic niche partitioning following successive invasions by fruit flies in La Réunion. Journal of Animal Ecology 75, 518-526.

Folk, D.G. \& Bradley, T.J. (2003) Evolved patterns and rates of water loss and ion regulation in laboratory-selected populations of Drosophila melanogaster. Journal of Experimental Biology 206, 2779-2786.

Folk, D.G. \& Bradley, T.J. (2004) The evolution of recovery from desiccation stress in laboratory-selected populations of Drosophila melanogaster. Journal of Experimental Biology 207, 2671-2678.
Folk, D.G., Han, C. \& Bradley, T.J. (2001) Water acquisition and partitioning in Drosophila melanogaster: effects of selection for desiccation-resistance. Journal of Experimental Biology 204, 3323-3331.

Gefen, E., Marlon, A.J. \& Gibbs, A.G. (2006) Selection for desiccation resistance in adult Drosophila melanogaster affects larval development and metabolite accumulation. Journal of Experimental Biology 209, 3293-3300.

Gibbs, A.G. \& Markow, T.A. (2001) Effects of age on water balance in Drosophila species. Physiological and Biochemical Zoology 74, 520-530.

Gibbs, A.G. \& Matzkin, L.M. (2001) Evolution of water balance in the genus Drosophila. Journal of Experimental Biology 204, 2331-2338.

Gibbs, A.G., Chippindale, A.K. \& Rose, M.R. (1997) Physiological mechanisms of evolved desiccation resistance in Drosophila melanogaster. Journal of Experimental Biology 200, 1821-1832.

Gibbs, A.G., Fukuzato, F. \& Matzkin, L.M. (2003) Evolution of water conservation mechanisms in Drosophila. Journal of Experimental Biology 206, 1183-1192.

Hancock, D.L., Hamacek, E.L., Lloyd, A.C. \& Elson-Harris, M.M. (2000) The Distribution and Host Plants of Fruit Flies (Diptera: Tephritidae) in Australia. Brisbane, Queensland, Department of Primary Industries.

Kaspi, R., Feitelson, I., Drezner, T. \& Yuval, B. (2001) A novel method for rearing the progeny of wild Mediterranean fruit flies using artificial fruit. Phytoparasitica 29, 15-22.

Kellermann, V., van Heerwaarden, B., Sgrò, C.M. \& Hoffmann, A.A. (2009) Fundamental evolutionary limits in ecological traits drive Drosophila species distributions. Science 325, 1244-1246.

Klassen, W. \& Curtis, C.F. (2005) History of the sterile insect technique. pp. 3-36 in Dyck, V.A., Hendrichs, J. \& Robinson, A.S. (Eds) Sterile Insect Technique: Principles and Practice in Area-wide Integrated Pest Management. Dordrecht, The Netherlands, Springer.

Kleynhans, E. \& Terblanche, J.S. (2009) The evolution of water balance in Glossina (Diptera: Glossinidae): correlations with climate. Biology Letters 5, 93-96.

Kleynhans, E. \& Terblanche, J.S. (2011) Complex interactions between temperature and relative humidity on water balance of adult tsetse (Glossinidae: Diptera): implications for climate change. Frontiers in Physiology 2, 1-10.

Knipling, E.F. (1959) Sterile-male method of population control. Science 130, 902-904.

Le Lagadec, M.D., Chown, S.L. \& Scholtz, C.H. (1998) Desiccation resistance and water balance in southern African keratin beetles (Coleoptera, Trogidae): the influence of body size and habitat. Journal of Comparative Physiology B 168, 112-122.

Marron, M.T., Markow, T.A., Kain, K.J. \& Gibbs, A.G. (2003) Effects of starvation and desiccation on energy metabolism in desert and mesic Drosophila. Journal of Insect Physiology 49, 261-270.

Matzkin, L.M., Watts, T.D. \& Markow, T.A. (2009) Evolution of stress resistance in Drosophila: interspecific variation in tolerance to desiccation and starvation. Functional Ecology 23, $521-527$.

Meats, A. (1989) Water relations of Tephritidae. pp. 241-246 in Robinson, A. \& Hooper, G. (Eds) Fruit Flies: Biology, Natural Enemies and Control. Rotterdam, Elsevier.

Meats, A., Holmes, H.M. \& Kelly, G.L. (2004) Laboratory adaptation of Bactrocera tryoni (Diptera: Tephritidae) decreases 
mating age and increases protein consumption and number of eggs produced per milligram of protein. Bulletin of Entomological Research 94, 517-524.

Nghiem, D., Gibbs, A.G., Rose, M.R. \& Bradley, T.J. (2000) Postponed aging and desiccation resistance in Drosophila melanogaster. Experimental Gerontology 35, 957-969.

Parkash, R., Aggarwal, D.D., Kalra, B. \& Ranga, P. (2011) Divergence of water balance mechanisms in two melanic Drosophila species from the western Himalayas. Comparative Biochemistry and Physiology A 158, 531-541.

Perez-Staples, D., Prabhu, V. \& Taylor, P.W. (2007) Post-teneral protein feeding enhances sexual performance of Queensland fruit flies. Physiological Entomology 32, 225-232.

Pérez-Staples, D., Weldon, C.W. \& Taylor, P.W. (2011) Sex differences in developmental response to yeast hydrolysate supplements in adult Queensland fruit fly. Entomologia Experimentalis et Applicata 141, 103-113.

Plant Health Australia (2008) Draft National Fruit Fly Strategy. Deakin, ACT, Australia, Plant Health Australia.

Rajpurohit, S., Parkash, R., Singh, S. \& Ramniwas, S. (2008) Climate change, boundary increase and elongation of a pre-existing cline: a case study in Drosophila ananassae. Entomological Research 38, 268-275.

R Development Core Team (2007) R: A Language and Environment for Statistical Computing. Vienna, Austria, R Foundation for Statistical Computing.

Sørensen, J.G., Addison, M.F. \& Terblanche, J.S. (2012) Massrearing of insects for pest management: challenges, synergies and advances from evolutionary physiology. Crop Protection 38, 87-94.

Telonis-Scott, M., Guthridge, K.M. \& Hoffmann, A.A. (2006) A new set of laboratory-selected Drosophila melanogaster lines for the analysis of desiccation resistance: response to selection, physiology and correlated responses. Journal of Experimental Biology 209, 1837-1847.

Terblanche, J.S., Clusella-Trullas, S. \& Chown, S.L. (2010) Phenotypic plasticity of gas exchange pattern and water loss in Scarabaeus spretus (Coleoptera: Scarabaeidae): deconstructing the basis for metabolic rate variation. Journal of Experimental Biology 213, 2940-2949.

Terras, M.A., Sundaralingam, S., Shaw, T., Cooke, B., Rice, J., Jessup, A.J. \& Dominiak, B.C. (1999) Fruit Fly Production Facility. Annual Report. 1998/99. Sydney, Tri-State Fruit Fly, NSW Agriculture.

Warburg, M.S. \& Yuval, B. (1997) Effects of energetic reserves on behavioral patterns of Mediterranean fruit flies (Diptera: Tephritidae). Oecologia 112, 314-319.

Weldon, C.W. \& Taylor, P.W. (2010) Desiccation resistance of adult Queensland fruit flies Bactrocera tryoni decreases with age. Physiological Entomology 35, 385-390.

Yonow, T. \& Sutherst, R.W. (1998) The geographical distribution of the Queensland fruit fly, Bactrocera (Dacus) tryoni, in relation to climate. Australian Journal of Agricultural Research 49, 935-953.

Yuval, B., Kaspi, R., Shloush, S. \& Warburg, M.S. (1998) Nutritional reserves regulate male participation in Mediterranean fruit fly leks. Ecological Entomology 23, 211-215. 